\title{
Carbon in aggregate size classes in a Rhodic Eutrudox under different cropping systems
}

\section{Carbono em classes de agregados em um Latossolo Vermelho sob diferentes sistemas de cultura}

\author{
Wesley Machado ${ }^{1 *}$; Thadeu Rodrigues de Melo'; Alex Figueiredo'; \\ José Victor Freitas dos Santos²; Felipe Gasparello Luccas ${ }^{3}$; Júlio Cézar Franchini"; \\ Maria de Fátima Guimarães ${ }^{5}$ J João Tavares Filho ${ }^{5}$
}

\begin{abstract}
Soil productive capacity is related to levels of carbon (C) in aggregates of different sizes. The aim of this study was to assess total organic carbon levels in different size classes of water-stable aggregates in a Rhodic Eutrudox under different production systems. The cropping systems assessed were no-tillage (NT); no-tillage scarified every three years (NTS); disk plowing (DP) and heavy disking (HD). All systems were subjected to crop succession (S) (soybean - Glycine max / wheat - Triticum aestivum) and rotation (R) (soybean, maize (Zea mays), wheat) and cover and green manure (Lupinus albus, Raphanus sativus and Avena strigosa). Intact soil samples were collected in trenches at depths of $0-0.10 ; 0.10$ $0.20 ; 0.20-0.30$ and $0.30-0.40 \mathrm{~m}$. The highest levels of carbon were found under no-tillage, irrespective of the aggregate size class. In all treatments, the top layer $(0.0-0,10 \mathrm{~m})$ under crop succession showed the highest carbon content for all aggregate size classes. However, at depths below $0.10 \mathrm{~m}$, crop rotation exhibited the highest carbon levels (between 12 and $20 \mathrm{~g} \mathrm{~kg}^{-1}$ ). After 29 years of trials, cropping systems with the lowest soil disturbance combined with crop rotation were found to contribute to raising the level of carbon in the soil and maintaining stable aggregates.
\end{abstract}

Key words: Aggregation. Cropping system. Production system. Structure.

\section{Resumo}

A capacidade produtiva do solo está relacionada a níveis de carbono (C) em agregados de diferentes tamanhos. O objetivo deste estudo foi avaliar os níveis totais de carbono orgânico em diferentes classes de tamanho de agregados estáveis em água em um Latossolo Vermelho eutroférrico sob diferentes sistemas de produção após 26 anos. Os sistemas de cultivo avaliados foram plantio direto (NT); Plantio direto escarificado a cada três anos (NTS); arado de disco (DP) e disco pesado (HD). Todos os sistemas foram submetidos a sucessão de culturas (S) (soja - Glycine max / trigo - Triticum aestivum)

\footnotetext{
1 Discentes, Curso de Doutorado do Programa de Pós-Graduação em Agronomia, Centro de Ciências Agrárias, Universidade Estadual de Londrina, UEL, Londrina, PR, Brasil. E-mail: w.machado@agronomo.eng.br; thadeurodrigues@hotmail.com; alexkdn@hotmail.com

2 Discente, Instituto Agronômico do Paraná, IAPAR, Londrina, PR, Brasil. E-mail: victorfreitas43@gmail.com

3 Discente, Curso de Graduação em Agronomia, Centro de Ciências Agrárias, UEL, Londrina, PR, Brasil. E-mail: felipegluccas@ gmail.com

4 Dr., Pesquisador, Empresa Brasileira de Pesquisa Agropecuária, EMBRAPA Soja, Centro Nacional de Pesquisa em Soja, Distrito de Warta, Londrina, PR, Brasil. E-mail: julio.franchini@embrapa.br

5 Profs. Drs., Departamento de Agronomia, Centro de Ciências Agrárias, UEL, Londrina, PR, Brasil. E-mail: mfatima@uel.br; tavares@uel.br

* Author for correspondence
} 
e rotação (R) (soja; milho (Zea mays); trigo) e de cobertura e adubação verde (tremoço (Lupinus albus), nabo forrageiro (Raphanus sativus) e aveia preta (Avena strigosa). As amostras de solo intactas foram coletadas em trincheiras em profundidades de 0 a 0,$10 ; 0,10-0,20 ; 0,20-0,30$ e $0,30-0,40 \mathrm{~m}$. Os níveis mais elevados de carbono foram encontrados em plantio direto, independentemente da classe de tamanho agregado. Em todos os tratamentos, a camada superior $(0,0-0,10 \mathrm{~m})$ sob sucessão de culturas mostrou os teores de carbono mais altos para todas as classes de tamanho agregado. No entanto, nas profundidades abaixo de $0,10 \mathrm{~m}$, a rotação das culturas exibiu os maiores níveis de carbono (entre $12 \mathrm{e}$ $20 \mathrm{~g} \mathrm{~kg}^{-1}$ ). Após 29 anos da implantação do experimento, os sistemas de cultivo com o menor distúrbio do solo combinados com a rotação das culturas contribuíram para elevar o nível de carbono no solo e manter agregados estáveis.

Palavras-chave: Agregação. Sistema de cultura. Sistemas de produção. Estrutura.

Organic matter is essential for ensuring the quality and productive capacity of the soil, since it affects the soil's chemical attributes (nutrient availability), physical attributes (aggregation and structure) and biological attributes (biological activity). However, in tropical regions the dynamics of organic matter are regulated by high temperatures and rainfall, resulting in a high rate of decomposition. Agricultural practices therefore directly affect additions and losses of organic matter and carbon in the soil, and therefore the formation and stability of aggregates (VEZZANI; MIELNICZUK, 2011).

The aim of this study was to assess the level of total organic carbon in different size classes of water-stable aggregate in a Rhodic Eutrudox under various cropping systems with succession and rotation after 26 years.

The experiment was set up in 1988/1989 in Londrina, state of Parana, $\left(23^{\circ} 11^{\prime} 39^{\prime \prime} \mathrm{S}\right.$ and $\left.51^{\circ} 10^{\prime} 40^{\prime} \mathrm{W}\right)$, in a Rhodic Eutrudox (EMBRAPA, 2013). Details of the experimental area can be found in Barreto et al. (2009).

The experimental design consisted of randomized blocks in a 2 x 4 factorial arrangement, with four replications, involving two production systems and four cropping systems. The two production systems assessed were: 1 - crop succession (S) and (soybean - Glycine max / wheat - Triticum aestivum) and 2 rotation (R) (soybean, maize (Zea mays), wheat) and cover and green manure (Lupinus albus, Raphanus sativus and Avena strigosa)The four cropping systems assessed were: no-tillage (NT); no-tillage with scarification every three years (NTS) in winter; conventional preparation by disk plowing (DP) and conventional preparation with heavy disking (HD).

Soil samples were collected from the $0.00-0.10$; $0.10-0.20 ; 0.20-0.30$ and $0.30-0.40 \mathrm{~m}$ layers. The method described in Claessen (1997), was used to determine the aggregate size classes and the carbon was determined after oxidation by $\mathrm{K}_{2} \mathrm{Cr}_{2} \mathrm{O}_{7}$ in sulfuric medium.

After testing the assumptions, the data were then subjected to analysis of variance and the means obtained were compared by non-orthogonal contrasts using the Scheffé test at 5 and $10 \%$ probability, to compare the cropping systems using SISVAR software.

Tilling intensity affected carbon levels, showing that cropping systems that induce soil disaggregation also cause a drop in carbon levels by exposing organic matter to bad weather, since the carbon is no longer physically protected inside the aggregates.

Working on four farming systems Costa Júnior et al. (2012) observed that NT boosted carbon levels in macro and microaggregates compared to conventional preparation (CP) systems and pasture, and in some cases the result were better than under savanna vegetation. The accumulation of TOC in larger aggregates could be due to the higher contribution of plant waste (both shoots and roots) resulting from the plant diversity involved in the production system, especially in the $0.00-0.20$ $\mathrm{m}$ layer with crop rotation under NT (Table 1 and 2 ). This build-up of carbon under NT is also due to 
continuous additions from plant waste, the absence of tillage and the system's consolidation time. This has also be reported by other authors (COSTA JÚNIOR et al., 2012).
In aggregates $>8 \mathrm{~mm}$, the use of soil preparation implements affected the level of TOC, with lower levels compared to conservationist practices, even under NTS with scarification every three years.

Table 1. Mean values of total organic carbon $\left(\mathrm{g} \mathrm{kg}^{-1}\right)$ through different screens and in different production systems (rotation and succession) and cropping systems (no-tillage; no-tillage with scarification; disk plowing and heavy disking), at four depths.

\begin{tabular}{|c|c|c|c|c|c|c|c|c|}
\hline \multicolumn{9}{|c|}{ Total Organic Carbon $\left(\mathrm{g} \mathrm{kg}^{-1}\right)(8 \mathrm{~mm})$} \\
\hline \multirow{3}{*}{$\begin{array}{l}\text { Production Systems } \\
\text { / Cropping Systems }\end{array}$} & \multicolumn{8}{|c|}{ Depths (m) } \\
\hline & \multicolumn{2}{|c|}{$0.00-0.10$} & \multicolumn{2}{|c|}{$0.10-0.20$} & \multicolumn{2}{|c|}{$0.20-0.30$} & \multicolumn{2}{|c|}{$0.30-0.40$} \\
\hline & Succession & Rotation & Succession & Rotation & Succession & Rotation & Succession & Rotation \\
\hline No Tillage & 23.18 & 20.64 & 16.85 & 16.75 & 12.85 & 13.83 & 9.88 & 12.95 \\
\hline No Tillage, Scarified & 17.14 & 18.31 & 14.8 & 13.71 & 12.07 & 11.27 & 7.72 & 11.93 \\
\hline Disk Plowing & 18.31 & 17.92 & 15.58 & 15.19 & 12.71 & 11.88 & 8.96 & 9.15 \\
\hline Heavy Disking & 9.25 & 16.07 & 14.41 & 16.94 & 10.9 & 8.76 & 5.66 & 3.4 \\
\hline \multicolumn{9}{|c|}{$(4 \mathrm{~mm})$} \\
\hline No Tillage & 22.25 & 19.32 & 14.41 & 15.38 & 11.49 & 12.46 & 9.25 & 13.12 \\
\hline No Tillage, Scarified & 18.31 & 17.53 & 15.19 & 15.01 & 17.71 & 8.96 & 7.4 & 5.06 \\
\hline Disk Plowing & 17.53 & 16.75 & 15.19 & 14.41 & 12.46 & 12.29 & 5.45 & 8.18 \\
\hline Heavy Disking & 15.19 & 15.77 & 13.05 & 13.14 & 9.54 & 7.3 & 6.03 & 8.18 \\
\hline \multicolumn{9}{|c|}{$(2 \mathrm{~mm})$} \\
\hline No Tillage & 20.79 & 18.4 & 13.83 & 14.61 & 9.93 & 12.46 & 7.79 & 12.31 \\
\hline No Tillage, Scarified & 17.53 & 16.75 & 14.02 & 14.8 & 11.49 & 9.83 & 5.84 & 6.82 \\
\hline Disk Plowing & 16.16 & 16.75 & 14.41 & 12.66 & 11.29 & 10.51 & 5.74 & 6.62 \\
\hline Heavy Disking & 16.16 & 15.1 & 13.24 & 11.39 & 4.57 & 8.03 & 6.6 & 5.18 \\
\hline \multicolumn{9}{|c|}{$(1 \mathrm{~mm})$} \\
\hline No Tillage & 20.69 & 18.45 & 13.93 & 14.61 & 9.93 & 11.68 & 7.6 & 10.55 \\
\hline No Tillage, Scarified & 15.97 & 17.14 & 12.27 & 13.34 & 9.83 & 8.96 & 6.42 & 6.22 \\
\hline Disk Plowing & 13.65 & 13.44 & 13.63 & 13.63 & 12.66 & 8.14 & 4.09 & 4.09 \\
\hline Heavy Disking & 15.77 & 14.51 & 11.78 & 9.62 & 8.78 & 5.99 & 5.25 & 4.28 \\
\hline \multicolumn{9}{|c|}{$(0,5 \mathrm{~mm})$} \\
\hline No Tillage & 19.91 & 17.53 & 12.86 & 13.83 & 8.37 & 10.9 & 7.01 & 9.74 \\
\hline No Tillage, Scarified & 15.97 & 16.36 & 12.85 & 13.63 & 9.64 & 9.15 & 6.62 & 6.03 \\
\hline Disk Plowing & 13.83 & 12.66 & 12.85 & 12.85 & 11.1 & 7.4 & 6.81 & 2.55 \\
\hline Heavy Disking & 15.19 & 14.99 & 12.07 & 8.83 & 5.84 & 4.96 & 4.67 & 3.31 \\
\hline \multicolumn{9}{|c|}{$(0,25 \mathrm{~mm})$} \\
\hline No Tillage & 19.96 & 17.14 & 12.27 & 13.05 & 8.18 & 10.51 & 5.84 & 9.83 \\
\hline No Tillage, Scarified & 15.58 & 15.97 & 14.02 & 12.85 & 9.35 & 8.96 & 7.11 & 5.06 \\
\hline Disk Plowing & 14.8 & 12.07 & 13.63 & 12.07 & 11.1 & 5.55 & 6.23 & 6.62 \\
\hline Heavy Disking & 16.75 & 14.21 & 11.05 & 9.1 & 9.35 & 6.76 & 7.4 & 5.45 \\
\hline
\end{tabular}

NT: no-tillage; NTS: no-tillage, scarified; DP: conventional preparation with disk plowing; HD: conventional preparation with heavy disking. 
As the soil is tilled, superficial carbon can be redistributed at depth, which can increase $\mathrm{C}$ levels in aggregates $<8 \mathrm{~mm}$, but according to Sarkhot et al. (2015), in the long term there is a drop in $\mathrm{C}$ as intensive tilling practices continue. However, using different plant species that accumulate higher quantities of dry matter in the soil, carbon tends to increase in aggregates in layers below $0.10 \mathrm{~m}$.
The explanation for the higher level of TOC is due to an increment on the surface from crop waste and the greater concentration of roots at depth, especial in the crop rotation system because of the diversity of plant species that exploit deeper soil layers, as well as the soluble organic compounds excreted by plants and microorganisms.

Table 2. Values of $p$ in non-orthogonal contrasts through different screens and with different production systems (rotation and succession) and cropping systems (no-tillage; no-tillage, scarified; disk plowing and heavy disking) at four depths

\begin{tabular}{|c|c|c|c|c|c|c|c|c|}
\hline \multicolumn{9}{|c|}{ Total Organic Carbon $\left(\mathrm{g} \mathrm{kg}^{-1}\right)(8 \mathrm{~mm})$} \\
\hline \multirow{3}{*}{$\begin{array}{l}\text { Production Systems } \\
\text { / Cropping Systems }\end{array}$} & \multicolumn{8}{|c|}{ Depths (m) } \\
\hline & \multicolumn{2}{|c|}{$0.00-0.10$} & \multicolumn{2}{|c|}{$0.10-0.20$} & \multicolumn{2}{|c|}{$0.20-0.30$} & \multicolumn{2}{|c|}{$0.30-0.40$} \\
\hline & Succession & Rotation & Succession & Rotation & Succession & Rotation & Succession & Rotation \\
\hline Contrasts & \multicolumn{8}{|c|}{$p>\mathrm{F}$ (Scheffé) } \\
\hline NT x DP & $0.002 *$ & $0.031 *$ & 0.2 & 0.124 & 0.821 & $0.014^{*}$ & 0.317 & $0.002 *$ \\
\hline NT x HD & $0.000^{*}$ & $0.002 *$ & $0.028^{*}$ & 0.835 & $0.014^{*}$ & $0.000^{*}$ & $0.001 *$ & $0.000 *$ \\
\hline NT x NTS & $0.000^{*}$ & $0.056^{*}$ & $0.054^{*}$ & $0.010^{*}$ & 0.247 & $0.003 *$ & $0.042 *$ & 0.27 \\
\hline DP $x$ HD & $0.000^{*}$ & 0.115 & 0.233 & $0.089 * *$ & $0.020^{*}$ & $0.001^{*}$ & $0.005^{*}$ & $0.000^{*}$ \\
\hline NTS x HD & $0.000^{*}$ & $0.065 * *$ & 0.679 & $0.007 *$ & $0.098 * *$ & $0.004 *$ & $0.039^{*}$ & $0.000 *$ \\
\hline NTS x DP & 0.297 & 0.719 & 0.415 & 0.141 & 0.34 & 0.361 & 0.213 & $0.012^{*}$ \\
\hline $\mathrm{CV} \%$ & \multicolumn{2}{|c|}{5.95} & \multicolumn{2}{|c|}{5.84} & \multicolumn{2}{|c|}{5.29} & \multicolumn{2}{|c|}{9.91} \\
\hline \multicolumn{9}{|c|}{$(4 \mathrm{~mm})$} \\
\hline NT x DP & $0.007 *$ & $0.082 * *$ & 0.516 & 0.42 & 0.301 & 0.222 & 0.16 & $0.075 * *$ \\
\hline NT x HD & $0.001 *$ & $0.025^{*}$ & 0.256 & $0.079 * *$ & $0.058^{* *}$ & $0.000^{*}$ & 0.229 & $0.075 * *$ \\
\hline NT x NTS & $0.016^{*}$ & 0.202 & 0.516 & 0.755 & $0.000^{*}$ & $0.004 *$ & 0.48 & $0.008^{*}$ \\
\hline DP x HD & 0.109 & 0.477 & $0.091 * *$ & 0.299 & $0.011 *$ & $0.002 *$ & 0.822 & 1.000 \\
\hline NTS x HD & $0.043^{*}$ & 0.213 & $0.091 * *$ & 0.135 & $0.000^{*}$ & $0.097 * *$ & 0.601 & 0.243 \\
\hline NTS x DP & 0.564 & 0.564 & 1.000 & 0.615 & $0.000^{*}$ & $0.029 *$ & 0.458 & 0.243 \\
\hline $\mathrm{CV} \%$ & \multicolumn{2}{|c|}{7.26} & \multicolumn{2}{|c|}{5.97} & \multicolumn{2}{|c|}{7.73} & \multicolumn{2}{|c|}{32.39} \\
\hline \multicolumn{9}{|c|}{$(2 \mathrm{~mm})$} \\
\hline NT x DP & $0.032 *$ & 0.402 & 0.599 & $0.097 * *$ & 0.582 & 0.435 & 0.366 & $0.022 *$ \\
\hline NT x HD & $0.032 *$ & 0.108 & 0.599 & $0.012 *$ & $0.046^{*}$ & $0.091 * *$ & 0.594 & $0.007 *$ \\
\hline NT x NTS & 0.113 & 0.402 & 0.86 & 0.86 & 0.53 & 0.297 & 0.388 & $0.027 *$ \\
\hline DP x HD & 1.000 & 0.403 & 0.301 & 0.264 & $0.016^{*}$ & 0.323 & 0.701 & 0.521 \\
\hline NTS x HD & 0.488 & 0.403 & 0.485 & $0.008^{*}$ & $0.014^{*}$ & 0.469 & 0.733 & 0.466 \\
\hline NTS x DP & 0.488 & 1.000 & 0.725 & $0.071 * *$ & 0.937 & 0.783 & 0.966 & 0.928 \\
\hline CV \% & \multicolumn{2}{|c|}{11.07} & \multicolumn{2}{|c|}{7.93} & \multicolumn{2}{|c|}{24.69} & \multicolumn{2}{|c|}{30.55} \\
\hline
\end{tabular}


continuation

\begin{tabular}{|c|c|c|c|c|c|c|c|c|}
\hline \multicolumn{9}{|c|}{$(1 \mathrm{~mm})$} \\
\hline NT x DP & $0.021^{*}$ & $0.084 * *$ & 0.856 & 0.55 & 0.161 & $0.076^{* *}$ & 0.162 & $0.018 *$ \\
\hline NT x HD & $0.090 * *$ & 0.164 & 0.2 & $0.008 *$ & 0.539 & $0.009 *$ & 0.339 & $0.021 *$ \\
\hline NT x NTS & 0.101 & 0.63 & 0.316 & 0.44 & 0.957 & 0.161 & 0.626 & $0.091 * *$ \\
\hline DP x HD & 0.441 & 0.694 & 0.265 & $0.026^{*}$ & $0.055^{*}$ & 0.261 & 0.629 & 0.936 \\
\hline NTS x HD & 0.943 & 0.342 & 0.762 & $0.037 *$ & 0.574 & 0.129 & 0.629 & 0.427 \\
\hline NTS x DP & 0.401 & 0.189 & 0.406 & 0.857 & 0.147 & 0.661 & 0.341 & 0.928 \\
\hline CV \% & \multicolumn{2}{|c|}{16.41} & \multicolumn{2}{|c|}{12.33} & \multicolumn{2}{|c|}{19.21} & \multicolumn{2}{|c|}{38.86} \\
\hline \multicolumn{9}{|c|}{$(0.5 \mathrm{~mm})$} \\
\hline NT x DP & $0.030^{*}$ & $0.073 * *$ & 0.997 & 0.53 & 0.242 & 0.14 & 0.934 & $0.009 *$ \\
\hline NT x HD & $0.081 * *$ & 0.327 & 0.613 & $0.006^{*}$ & 0.276 & $0.020^{*}$ & 0.328 & $0.016^{*}$ \\
\hline NT x NTS & 0.137 & 0.646 & 0.998 & 0.899 & 0.578 & 0.444 & 0.868 & 0.132 \\
\hline DP x HD & 0.592 & 0.364 & 0.615 & $0.021 *$ & $0.035^{*}$ & 0.294 & 0.368 & 0.748 \\
\hline NTS x HD & 0.759 & 0.592 & 0.615 & $0.008 *$ & 0.112 & $0.083 * *$ & 0.412 & 0.257 \\
\hline NTS x DP & 0.404 & 0.161 & 1.000 & 0.615 & 0.522 & 0.444 & 0.934 & 0.928 \\
\hline $\mathrm{CV} \%$ & \multicolumn{2}{|c|}{15.68} & \multicolumn{2}{|c|}{12.09} & \multicolumn{2}{|c|}{26.32} & \multicolumn{2}{|c|}{39.2} \\
\hline \multicolumn{9}{|c|}{$(0.25 \mathrm{~mm})$} \\
\hline NT x DP & $0.057 * *$ & $0.061 * *$ & 0.429 & 0.57 & $0.027 *$ & $0.004^{*}$ & 0.729 & $0.013^{*}$ \\
\hline NT x HD & 0.214 & 0.256 & 0.479 & 0.036 & 0.419 & $0.020^{*}$ & 0.181 & $0.002 *$ \\
\hline NT x NTS & $0.099 * *$ & 0.642 & 0.314 & 0.909 & 0.419 & 0.286 & 0.269 & $0.001 *$ \\
\hline DP x HD & 0.442 & 0.399 & 0.147 & $0.100 * *$ & 0.12 & 0.4 & 0.308 & 0.308 \\
\hline NTS x HD & 0.642 & 0.488 & $0.100 * *$ & $0.044^{*}$ & 1.000 & 0.142 & 0.8 & 0.729 \\
\hline NTS x DP & 0.756 & 0.138 & 0.819 & 0.648 & 0.12 & $0.031 *$ & 0.436 & 0.928 \\
\hline $\mathrm{CV} \%$ & \multicolumn{2}{|c|}{15.49} & \multicolumn{2}{|c|}{13.59} & \multicolumn{2}{|c|}{15.87} & \multicolumn{2}{|c|}{16.39} \\
\hline
\end{tabular}

*Scheffé test at $5 \%$;* Scheffé test at $10 \%$. NT: no-tillage; NTS: no-tillage, scarified; DP: conventional preparation with disk plowing; HD: conventional preparation with heavy disking.

In many cases, this response is not merely related to the quantity of organic matter deposited on the soil, but also to the volume and distribution of roots along the profile, and the system adopted, whether succession or rotation (QIAO et al., 2015). Planting different crops causes a build-up of carbon and enhances aggregate stability. Santos et al. (2012) observed that the different crops studied raised average $C$ levels in the $0.00-0.10 \mathrm{~m}$ layer, in line with our findings.

Increasing organic $\mathrm{C}$ levels results in an increase in soil aggregation indices by decreasing classes of lower diameter $(<0.25 \mathrm{~mm})$ (ASSIS et al., 2006; BATISTA et al., 2013). The TOC levels in microaggregates can be explained by the build- up of humic fractions, and in macroaggregates by free organic matter (particulate carbon). The accumulation of free organic matter is therefore linked to the formation of larger aggregates, and association with iron oxides helps form larger aggregates of larger diameter, enhancing carbon protection since the aggregation also depends on oxides (FERREIRA et al., 2007).

NT and NTS increased total organic carbon, irrespective of aggregate size, and especially in the $0.0-0.20 \mathrm{~m}$ layer. The highest carbon accumulations were observed at a depth of 0.0-0.10 m, and crop succession resulted in the highest average carbon level for all aggregate sizes. At lower depths, the highest average carbon levels were found under 
crop rotation. Heavy disking (HD) resulted in lower carbon levels, especially in aggregates $<8 \mathrm{~mm}$ in layers below $0.20 \mathrm{~m}$.

\section{Acknowledgements}

Our thanks to "Coordenação de Aperfeiçoamento de Pessoal de Nível Superior" and "Conselho Nacional de Desenvolvimento Científico e Tecnológico" for financial support, and also to Embrapa-Soja.

\section{References}

ASSIS, C. P.; JUCKSCH, I.; MENDONÇA, E. S.; NEVES, J. C. L. Carbono e nitrogênio em agregados de Latossolo submetido a diferentes sistemas de uso e manejo. Pesquisa Agropecuária Brasileira, Brasília, v. 41, n. 3, p. 1541-1550, 2006.

BATISTA, J.; PEREIRA, M.G.; CORREIA, M. E. F.; BIELUCZYK, W.; SCHIAVO, J. A.; ROWS, J. R. C. Teores e estoque de carbon em frações lábeis e recalcitrantes da matéria orgânica do solo sob integração lavoura-pecuária no bioma Cerrado. Semina: Ciências Agrárias, Londrina, v. 34, n. 4, p. 3377-3388, 2013. DOI: 10.5433/1679-0359.2013v34n6Sup11p3377

BARRETO, R. C.; MADARI, B. E.; MADDOCK, J. E.; MACHADO, P. L. O. A.; TORRES, E.; FRANCHINI, J. C.; COSTA, A. R. The impact of soil management on aggregation, carbon stabilization and carbon loss as $\mathrm{CO}_{2}$ in the surface layer of a Rhodic Ferrasol in Southern Brazil. Agriculture, Ecosystems and Environment, Amsterdam, v. 132, n. 5, p. 243-251, 2009. DOI: 10.1016/j.agee.2009.04.008

CLAESSEN, M. E. C. (Org.). Manual de métodos de análise de solos. 2. ed. Rio de Janeiro: Serviço Nacional de Levantamento e Conservação de Solos, 1997. 212 p.

COSTA JÚNIOR, C.; PÍCCOLO, M. D. C.; SIQUEIRA NETO, M.; BARBOSA de CAMARGO, P.; CERRI, C. C.; BERNOUX, M. Carbono em agregados do solo sob vegetação nativa, pastagem e sistemas agrícolas no bioma Cerrado. Revista Brasileira de Ciência do Solo, Viçosa, MG, v. 36, n. 5, p. 1311-1321, 2012.

EMPRESA BRASILEIRA DE PESQUISA AGROPECUÁRIA - EMBRAPA. Sistema Brasileiro de classificação de solos. 3. ed. Brasília: Editora Embrapa SPI, 2013.
FERREIRA, F. P.; AZEVEDO, A. C.; DALMOLIN, R. S. D.; GIRELLI, D. Carbono orgânico, óxidos de ferro e distribuição de agregados em dois solos derivados de basalto no Rio Grande do Sul - Brasil. Ciência Rural, Santa Maria, v. 37, n. 2, p. 381-388, 2007.

QIAO, Y.; MIAO, S.; LI, N.; XU, Y.; HAN, X.; ZHANG, B. Crop species affect soil organic carbon turnover in soil profile and among aggregate sizes in a Mollisol as estimated from natural ${ }^{13} \mathrm{C}$ abundance. Plant Soil, The Hague, v. 392, n. 8, p. 163-174, 2015. DOI: $10.1007 /$ s11104-015-2414-8

SANTOS, G. G.; SILVEIRA, P. M.; MARCHÃO, R. L.; PETTER, F. A.; BECQUER, T. Atributos químicos e estabilidade de agregados sob diferentes culturas de cobertura em Latossolo do Cerrado. Revista Brasileira de Engenharia Agrícola e Ambiental, Campina Grande, v. 16, n. 6, p. 1171-1178, 2012.

SARKHOT, D. V.; COMERFORD, N. B.; JOKELA, E. $\mathrm{J}$. Effects of forest management intensity on carbon and nitrogen content in different soil size fractions of a North Florida Spodosol. Plant Soil, The Hague, v. 294, n. 3, p. 291-303, 2007. DOI: 10.1007/s11104-007-9255-z

VEZZANI, F. M.; MIELNICZUK, J. Agregação e estoque de carbono em argissolo submetido a diferentes práticas de manejo agrícola. Revista Brasileira de Ciência do Solo, Viçosa, MG, v. 35, n. 2, p. 213-223, 2011. 